\title{
Flow e Engajamento no Trabalho: Associações com Recursos Individuais e Contexto Laboral
}

\author{
Larissa Sanford Ayres Farina ${ }^{1}$, Gabriel Dos Reis Rodrigues, Natália Kluwe Fagundes \\ Universidade Federal do Rio Grande do Sul, Porto Alegre-RS, Brasil \\ Thamires Casarotto Carafini \\ Universidade Luterana do Brasil, Canoas-RS, Brasil \\ Laisla Gabriele Conceição Soares Moreira \\ Centro Universitário FADERGS, Porto Alegre-RS, Brasil \\ Wagner De Lara Machado \\ Pontifícia Universidade Católica do Rio Grande do Sul, Porto Alegre-RS, Brasil \\ Claudio Simon Hutz \\ Universidade Federal do Rio Grande do Sul, Porto Alegre-RS, Brasil
}

\section{RESUMO}

As vivências de flow e engajamento se relacionam com atributos pessoais e laborais, gerando resultados superiores nos negócios, no desempenho dos trabalhadores e na satisfação de vida. Este estudo visou investigar flow e engajamento no trabalho e suas correlações com características pessoais e laborais. A amostra foi formada por 317 participantes (66,9\% do sexo feminino) com idade média de 39,72 anos $(D P=10,8)$, que responderam um questionário sociodemográfico e escalas que permitiram medir as variáveis alvo. Para análise de dados, foi realizada análise de rede. Os dados evidenciaram que alguns recursos individuais e do trabalho estão fortemente correlacionados com flow e engajamento laborais. Os resultados apontam para a importância de se promover um ambiente de trabalho que estimule o alinhamento de habilidades e a comunicação, além da diminuição de afetos negativos, para se potencializar as vivências de flow e engajamento nesse contexto.

Palavras-chave: flow; engajamento no trabalho; recursos pessoais; recursos do trabalho; demandas do trabalho.

ABSTRACT - Flow and Engagement at Work: Associations with Personal Resources and Work Environment

The experience of flow and engagement has been related to personal and work attributes, generating superior results related to business success, employee performance and life satisfaction. This study aimed to investigate flow and engagement at work and their associations with personal and work characteristics. The sample consisted of 317 participants (66.9\% female) with a mean age of 39.72 years $(S D=10.8)$, who responded to a socio-demographic questionnaire and scales that allowed the target variables to be measured. A network analysis was performed to interpret the data. The data showed that some individual and work resources are strongly correlated with work flow and engagement. The results indicate the importance of promoting a work environment that stimulates skills alignment, communication and the reduction of negative affect to enhance the flow and engagement experiences in this context. Keywords: Flow; work engagement; personal resources; work resources; job demands.

\section{RESUMEN - Flow y Compromiso con el Trabajo: Asociaciones con los Recursos Individuales y el Contexto Laboral}

Las vivencias de flow y compromiso están relacionadas con los atributos personales y laborales, generando resultados superiores en los negocios, en el rendimiento de los trabajadores y en la satisfacción con la vida. Este estudio tuvo como objetivo investigar el flow y el rendimiento en el trabajo y sus vínculos con las características personales y laborales. La muestra se compuso por 317 participantes (66.9\% mujeres) con una edad media de 39.72 años $(D S=10.8)$, que respondieron a un cuestionario sociodemográfico y a escalas que permitieron medir las variables objetivas. Para el análisis de datos, se realizó un análisis de red. Los datos mostraron que algunos recursos individuales y laborales están fuertemente correlacionados con el flow y el compromiso de trabajo. Los resultados señalan la importancia de promover un ambiente de trabajo que estimule la alineación de las habilidades y la comunicación; además de la reducción de afectos negativos, para potenciar el flow y las de compromiso en este contexto.

Palabras clave: flow; compromiso con el trabajo; recursos personales; recursos laborales, demandas laborales.

Diante da análise da literatura existente (Farina, Rodrigues, \& Hutz, 2018), estudos apontam que um maior conhecimento sobre flow e engajamento no trabalho pode ser utilizado pelas empresas para se gerar resultados superiores nos negócios, no desempenho individual de cada trabalhador e no seu bem-estar (Bakker, 
2008; Farina et al., 2018; Schaufeli, Dijikstra, \& Vazquez, 2013). No presente estudo, o construto engajamento será definido como um estado mental disposicional de investimento de energia, enfrentamento das dificuldades e direcionamento dos esforços individuais nas atividades laborais (Schaufeli, 2013), ou seja, um atributo pessoal positivo e independente relacionado ao trabalho, caracterizado por vigor, dedicação e concentração (Vazquez, Magnan, Pacico, Hutz, \& Schaufeli, 2015). Já o flow laboral diz respeito a um estado de consciência em que um indivíduo está totalmente ensimesmado e entusiasmado ao realizar uma determinada tarefa (Bakker, 2008; Csikszentmihalyi, 1999). O flow no trabalho é composto por: absorção (total imersão em uma tarefa), envolvimento no trabalho (prazer) e motivação intrínseca (Bakker, 2008).

Pesquisas apontam uma correlação entre os recursos e demandas laborais com engajamento, flow e melhor performance no ambiente organizacional (Mäkikangas, Bakker, Aunola, \& Demerouti, 2010; Schaufeli, 2013). Desse modo, torna-se interessante notar que, mesmo diferentes em cada organização, recursos de trabalho parecem influenciar nas vivências de flow e de engajamento (Schaufeli, 2013). Dessa forma, o entendimento dessas associações entre recursos laborais, flow e engajamento pode servir de base para se levantar oportunidades de desenvolvimento individual ou organizacional (Salanova, Bakker, \& Llorens, 2006).

As características do contexto laboral podem ser estudadas a partir do Modelo de Recursos e Demandas do Trabalho (Modelo RDT) (Taris \& Schaufeli, 2016). Esse paradigma é uma proposição relevante para se entender e nomear possíveis obstáculos e catalisadores do vínculo colaborador/organização (Taris \& Schaufeli, 2016). O Modelo RDT foi apresentado conceitualmente a partir de dois fatores: os recursos e as demandas laborais. Os recursos do trabalho dizem respeito aos aspectos físicos, psíquicos e sociais que diminuem as solicitações excessivas feitas pelas empresas aos seus colaboradores. Já as demandas laborais são as características físicas, psíquicas e sociais capazes de produzir esforço extra, facilitando o aparecimento de estresse excedente ao profissional. De acordo com esse paradigma, a disposição física e motivação do trabalhador advêm de um equilíbrio entre os recursos e as demandas laborais. Demandas excessivas podem gerar estresse, enquanto que os recursos colaboram para a experiência de flow e de engajamento (Cousins et al., 2004; Nielsen \& Cleal, 2010; Schaufeli, Bakker, \& Rhenen, 2009; Vazquez et al., 2015).

O presente estudo focará nos seguintes oito recursos de trabalho: suporte do líder, suporte dos colegas, clareza de papel, comunicação, desafios, justiça no trabalho, perspectiva de crescimento e alinhamento de habilidades, de acordo com as dimensões encontradas no estudo de construção e validação do Questionário de Recursos e Demandas do Trabalho de Farina et al. (no prelo). O suporte do líder está relacionado a quanto o líder estimula e articula sua equipe, entusiasmando, comprometendo e criando um clima de colaboração. O suporte dos colegas está ligado a quanto os profissionais percebem que as pessoas com cargos análogos aos seus os respaldam e estão dispostos para auxiliá-los ou contribuir em suas atividades. A clareza de papel está associada à percepção clara do colaborador de suas responsabilidades e comportamentos desejáveis dentro da instituição conjuntamente ao sentimento de que as suas tarefas cumpridas são importantes para a sua vida, para o serviço de seus colegas e de outros. A comunicação diz respeito às informações disponibilizadas ao colaborador sobre o funcionamento da organização (Farina et al., no prelo).

Os desafios versam sobre o nivelamento entre as capacidades do indivíduo e as solicitações da empresa. A justiça no trabalho refere-se ao tratamento legítimo e apropriado por parte dos pares, da organização e dos dirigentes nas relações de trabalho. Isso inclui uma carga de trabalho adequada além de exigências e procedimentos bem alinhados. A perspectiva de crescimento está associada à compreensão de que o trabalho pode promover o desenvolvimento e o conhecimento das pessoas. Por fim, o alinhamento de habilidades aborda a consciência de que o cargo assumido e suas responsabilidades estão de acordo com o que o funcionário pode entregar devido as suas capacidades e habilidades pessoais. (Farina et al., no prelo).

Ainda, pesquisas indicam que os recursos individuais dos profissionais também estão relacionados ao flow e ao engajamento laborais (Datu \& Mateo, 2015; Salanova et al., 2006). Recursos individuais são autoavaliações positivas a respeito da habilidade de controlar o ambiente e fazer a diferença em seu entorno. Pesquisas sobre recursos individuais verificaram que variáveis como autoeficácia, esperança e bem-estar podem ser antecedentes da experiência de flow no trabalho (Datu \& Mateo, 2015; Salanova et al., 2006; Zubair \& Kamal, 2015). Diante disso, torna-se relevante investigar as relações entre flow e engajamento no trabalho e suas associações com os recursos individuais.

No presente estudo, os recursos individuais serão representados pela autoeficácia, esperança, afetos positivos e negativos, e satisfação com a vida. Entende-se por autoeficácia a crença que uma pessoa tem sobre suas competências e capacidades de controlar e impactar com sucesso o seu entorno, o que não necessariamente estaria ligada à realidade em si (Pacico, Ferraz, \& Hutz, 2014; Salanova et al., 2006). No ambiente laboral, uma forte crença de eficácia pode impactar positivamente o bem-estar do trabalhador, facilitando as vivências de flow e engajamento laboral (Consiglio, Borgogni, Di Tecco, \& Schaufeli, 2016).

Da mesma forma, a esperança tem se mostrado positivamente associada com flow e com engajamento (Uusiautti \& Määttä, 2015; Zubair \& Kamal, 2015). 
A esperança pode ser entendida como uma série de pensamentos voltados a uma meta, compostos por rotas (criação de caminhos que levam ao que se deseja) e agenciamento (motivações para se usar as rotas) (Uusiautti \& Määttä, 2015). Funcionários esperançosos possivelmente são mais propícios a vigorosamente buscar seus objetivos e a criar alternativas diante de obstáculos, características também presentes nas pessoas que vivenciam flow e são engajadas (Zubair \& Kamal, 2015).

Já a satisfação de vida pode ser definida como o grau de entusiasmo e prazer que uma pessoa percebe sobre sua vida (Hutz, Zanon, \& Bardagi, 2014). Assim, esse recurso individual é uma avaliação subjetiva, feita durante uma autorreflexão sobre os aspectos da vida em geral que se inclina a ser estável com o passar dos anos (Hutz et al., 2014). Datu e Mateo (2015) apontaram correlações positivas entre satisfação com a vida e flow no trabalho. Ainda, Hakanen e Schaufeli (2012) indicaram que colaboradores engajados demonstram mais emoções e atitudes positivas, o que pode contribuir para a sua satisfação com a vida.

O objetivo geral desse estudo é investigar as associações entre os conceitos de flow e engajamento no trabalho com características pessoais e recursos de trabalho. Os objetivos específicos dessa pesquisa são estudar as relações entre recursos individuais com flow e engajamento laborais; analisar a relação entre os recursos e demandas do trabalho com flow e engajamento laborais. Desse modo, este estudo identifica como os recursos individuais e laborais estão relacionados ao engajamento e ao flow no trabalho. Ao se examinar como essas conexões acontecem, pode-se pensar em intervenções eficazes que influenciam e potencializam a vivência e experiência de flow e de engajamento no ambiente laboral. Eventualmente, por exemplo, esta pesquisa poderia servir como base para um programa de desenvolvimento de lideranças ou de equipes.

\section{Método}

\section{Participantes}

Os seguintes critérios de inclusão foram levados em consideração: residir no Brasil, ter entre 18 e 65 anos de idade e realizar uma atividade profissional, com ou sem vínculo empregatício. A amostra por conveniência foi formada por 317 participantes, a maioria do sexo feminino $(n=212 ; 66,9 \%)$, e as idades variando entre 20 e 65 anos $(M=39,72, D P=10,8)$. A maior parte dos indivíduos era casado $(n=146 ; 46,1 \%)$. Os respondentes afirmaram residir em 19 estados brasileiros, abrangendo todas as regiões do Brasil, sendo a maioria do Rio Grande do Sul $(n=228 ; 71,9 \%)$. A escolaridade mínima observada foi de ensino médio completo e a maioria declarou possuir pós-graduação $(n=216 ; 68,1 \%)$.

\section{Instrumentos}

Os participantes responderam a um questionário sociodemográfico para coleta de dados como sexo, idade, escolaridade etc. Além desse, foram utilizados os instrumentos apresentados a seguir. As médias, desvios padrão e confiabilidade desses instrumentos obtidas com a amostra deste estudo estão no material suplementar $\mathrm{A}^{2}$.

A Versão Brasileira da Work-Related Flow Inventory (WOLF), de Freitas, Damásio, e Koller (2019), trata-se de um escala de treze itens, tipo Likert de seis pontos. A análise fatorial confirmatória apresentou três fatores como uma solução adequada para a escala. O participante deve marcar a frequência com que vivencia o estado descrito no item, entre "0" (nunca) e "6" (todo dia). Exemplos de itens para cada fator: absorção, "Quando estou trabalhando, eu esqueço de tudo ao meu redor"; envolvimento, "Eu faço meu trabalho com muito prazer"; motivação intrínseca, "Quando estou trabalhando em alguma coisa, estou fazendo isso por mim mesmo(a)". Na validação para o Brasil de Freitas, Damásio e Koller (2019), os coeficientes de confiabilidade das três dimensões de WOLF foram avaliados por meio da confiabilidade composta. A dimensão absorção mostrou o valor de confiabilidade de 0,93 , a dimensão envolvimento no trabalho de 0,98 e a dimensão motivação intrínseca de 0,87 .

O engajamento no trabalho foi medido por meio da versão brasileira da Utrecht Work Engagement Scale UWES (Vazquez et al., 2015). Essa escala contém dezessete itens, tipo Likert de 7 pontos. Análises fatoriais resultaram em soluções adequadas tanto para um fator quanto para três fatores. O respondente deve marcar a frequência de sentimentos em relação ao trabalho, entre "0" (nunca) e "6" (sempre). Exemplos de itens para cada fator: vigor, "No meu trabalho, sinto que estou cheio de energia."; dedicação, "Sou entusiasmado com meu trabalho."; concentração: "Eu fico absorvido com meu trabalho". A consistência interna para o fator geral foi de $\alpha=0,95$; e, para cada dimensão: vigor: $\alpha=0,86$, dedicação: $\alpha=0,87$, e concentração: $\alpha=0,85$.

A autoeficácia foi mensurada por meio da Escala de Autoeficácia Geral de Pacico et al. (2014), uma escala de 20 itens tipo Likert de cinco pontos. Seu objetivo é medir o quanto o sujeito crê em sua capacidade de alcançar metas pessoais. O participante deverá marcar de "1" ("sempre falsa") a "5" ("sempre verdadeira") de acordo com o item (e.g., "Quando faço planos, sei que posso fazer que eles deem certo.”). Análises fatoriais demonstraram uma estrutura unidimensional com consistência interna adequada $(\alpha=0,89)$.

Com a intenção de se medir a esperança, foi utilizada a Escala de Esperança Disposicional de Pacico, Zanon, Bastianello, Reppold, e Hutz (2013). Os autores

${ }^{2}$ Material suplementar A: https://drive.google.com/file/d/1s0-cSpjOlKxpA4RoU0VfGQ6XyrNnrmdU/view? usp=sharing 
propõem uma estrutura unifatorial para a escala Likert de cinco pontos. Em 12 frases como "Eu me esforço para atingir meus objetivos.", o indivíduo deve marcar "5" quando a alternativa é "totalmente verdadeira" e "1" quando ela é "totalmente falsa". O coeficiente $\alpha$ foi de 0,79 na amostra de adultos, demonstrando consistência interna adequada da escala.

Foi utilizada a Escala de Afetos Positivos e Negativos (PANAS) desenvolvida e validada por Zanon, Bastianello, Pacico e Hutz (2013) para se medir os afetos positivos e negativos. A escala é tipo Likert de 5 pontos (de " 1 - Nem um pouco" a "5 - Extremamente") formada por 20 itens. Construída a partir de adjetivos, dez itens descrevem sentimentos e emoções contemplando "afetos positivos" (e.g., "amável"), e dez itens contemplando "afetos negativos" (e.g., "aflito"). Resultados indicaram consistência interna satisfatória, com $\alpha=0,83$ para afetos positivos e $\alpha=0,77$ para os negativos.

Para medir a satisfação com a vida, foi utilizada a Satisfaction with Life Scale, adaptada para o Brasil por Zanon, Bardagi, Layous e Hutz (2014). Trata-se de uma escala de cinco itens tipo Likert de sete pontos, no qual o participante assinala "7" se "concorda plenamente" com a afirmativa e "1" se "discordar plenamente". Exemplo de item: "A minha vida está próxima do meu ideal.". A unidimensionalidade da escala foi averiguada a partir de uma análise fatorial, sendo o coeficiente $\alpha=0,87$.

Para se medir os recursos e demandas do trabalho foi utilizado o Questionário de Recursos e Demandas do Trabalho - QRDT (Farina et al., no prelo). O instrumento contém 44 itens, tipo Likert de 4 pontos, e se propõe a avaliar oito dimensões do ambiente de trabalho percebido por trabalhadores entre 18 e 65 anos. O participante pode também responder "Não se aplica" caso o item não corresponda à sua realidade. Por exemplo, itens do fator Suporte do Líder não foram respondidos por alguns trabalhadores autônomos. Oito fatores foram extraídos utilizando-se uma Análise Fatorial Exploratória; seis dos oito obtiveram $\alpha>0,80$.

\section{Procedimentos}

A coleta de dados foi realizada por meio eletrônico, utilizando-se o software de serviços de desenvolvimento de pesquisa online SurveyMonkey. Nesse programa foram inseridos os questionários e escalas mencionados, que foram acessados por um link por ele fornecido. Em um único acesso o participante respondeu a todos os instrumentos. A pesquisa foi divulgada e disponibilizada para resposta em diferentes meios, como redes sociais, e-mails e/ou convite pessoal em locais de trabalho no Brasil.

\section{Análise de dados}

Para investigar as associações entre as variáveis investigadas foram conduzidas análises de rede (Schmittmann et al., 2013). A análise de rede é uma técnica exploratória de aprendizagem máquina que tem por objetivo estimar as associações parciais (condicionadas) entre variáveis (Machado, Vissoci, \& Epskamp, 2015). Esse método permite modelar um conjunto grande de variáveis e a relação entre elas, destacando aquelas com maior influência. Em uma primeira etapa, são estimadas as correlações parciais por meio da inversa da matriz de variância e covariância (Epskamp \& Fried, 2018). A seguir é aplicada uma regularização (penalização) do tipo Least Abssolute Shrinkage and Selection Operator (LASSO) a fim de fixar em zero os valores muito pequenos nas associações parciais (Friedman, Hastie, \& Tibshirani, 2008). A regularização tem por objetivo manter os parâmetros do modelo exploratório com maior probabilidade de representação dos parâmetros populacionais, evitando assim a apresentação de parâmetros falsos-positivos. A escolha do grafo com melhor proporção entre falsos-positivos e falsos-negativos se faz a partir do ajuste Extended Bayesian Criterion (EBIC) para gerar o grafo com menor resíduo (Foygel \& Drton, 2010).

$\mathrm{Na}$ segunda etapa da análise, a matriz de correlações parciais regularizada é representada por meio de um objeto gráfico (grafo) bidimensional no qual as variáveis são representadas por vértices (nodos) e suas relações por meio de arestas (linhas). A relação entre dois nodos é visualmente representada pela aresta que os liga, tanto pela sua espessura (e.g., arestas mais finas representam associações de menor magnitude) quanto pela sua cor (i.e., arestas azuis indicando associações positivas e, vermelhas, negativas). Assim, um nodo influencia todos os nodos ligados a ele, e vice-versa, conforme a magnitude dessa ligação (espessura das arestas) e a direção dessa relação (positiva ou negativa). A maior ativação em um nodo, portanto, pode levar ao aumento (caso a aresta seja positiva) ou à diminuição (caso a aresta seja negativa) dos outros nodos presentes no grafo. As análises foram conduzidas por meio do software R, em uma interface de uso chamada RStudio, e do pacote qgraph (Epskamp, Cramer, Waldorp, Schmittmann, \& Borsboom, 2012; RStudio, 2012).

É possível que dois nodos não estejam diretamente associados, mas que um terceiro nodo esteja no caminho mais curto entre essas duas variáveis. Considerando o grande número de nodos no presente modelo e para maior clareza na apresentação dos resultados, utilizou-se um gráfico de fluxo (flowchart) com o objetivo de ressaltar os vértices que possuem associação direta e associação indireta com o engajamento e com flow no trabalho. Ressalta-se que o vértice entre um nodo e outro evidencia associação, não causalidade. $\mathrm{O}$ grafo de rede, em menor proporção, é apresentado junto ao gráfico de fluxo.

A representação gráfica das relações entre as variáveis permite estimar medidas descritivas a respeito da importância de cada vértice no modelo, chamadas de medidas de centralidade. Considerando a presença tanto de arestas positivas quanto de arestas negativas no modelo, optou-se por estimar a medida de influência esperada (Robinaugh, Millner, \& McNally, 2016). Nessa medida, somam-se todas as arestas de um vértice de primeiro 
(associação direta) e segundo grau (associação indireta). Quanto maior o valor da medida de influência esperada, maior a probabilidade deste nodo, quando ativado, de ativar os nodos adjacentes. Deste modo, essa medida auxilia na identificação de nodos influentes e possíveis alvos de intervenção.

\section{Resultados}

Análises de correlação ${ }^{3}$ identificaram uma associação de alta magnitude entre flow e engajamento, sendo $r=0,85, p<001$. Por conta desta alta correlação, quando inseridas no mesmo modelo, as correlações parciais entre as demais variáveis foram praticamente nulas. Assim, optou-se por estimar dois modelos - um sem flow, o outro sem engajamento.
O modelo estimado para o flow, recursos individuais, e recursos e demandas de trabalho está apresentado na Figura 1. Nota-se que flow no trabalho apresentou associação direta e positiva com alinhamento de habilidades e afetos positivos. O flow também esteve diretamente e positivamente associado com as variáveis perspectiva de crescimento e desafios, porém de maneira menos intensa. Em relação aos recursos individuais, além dos afetos positivos, a variável autoeficácia apresentou associação direta e positiva com flow, e uma associação de maior magnitude com esperança. Ainda observando a Figura 1, as variáveis justiça, suporte do líder e afetos negativos apresentam-se apenas indiretamente associadas aoflow no trabalho. Os afetos negativos apresentaram associação indireta com flow, estando associada direta e negativamente com satisfação de vida, afetos positivos e esperança.

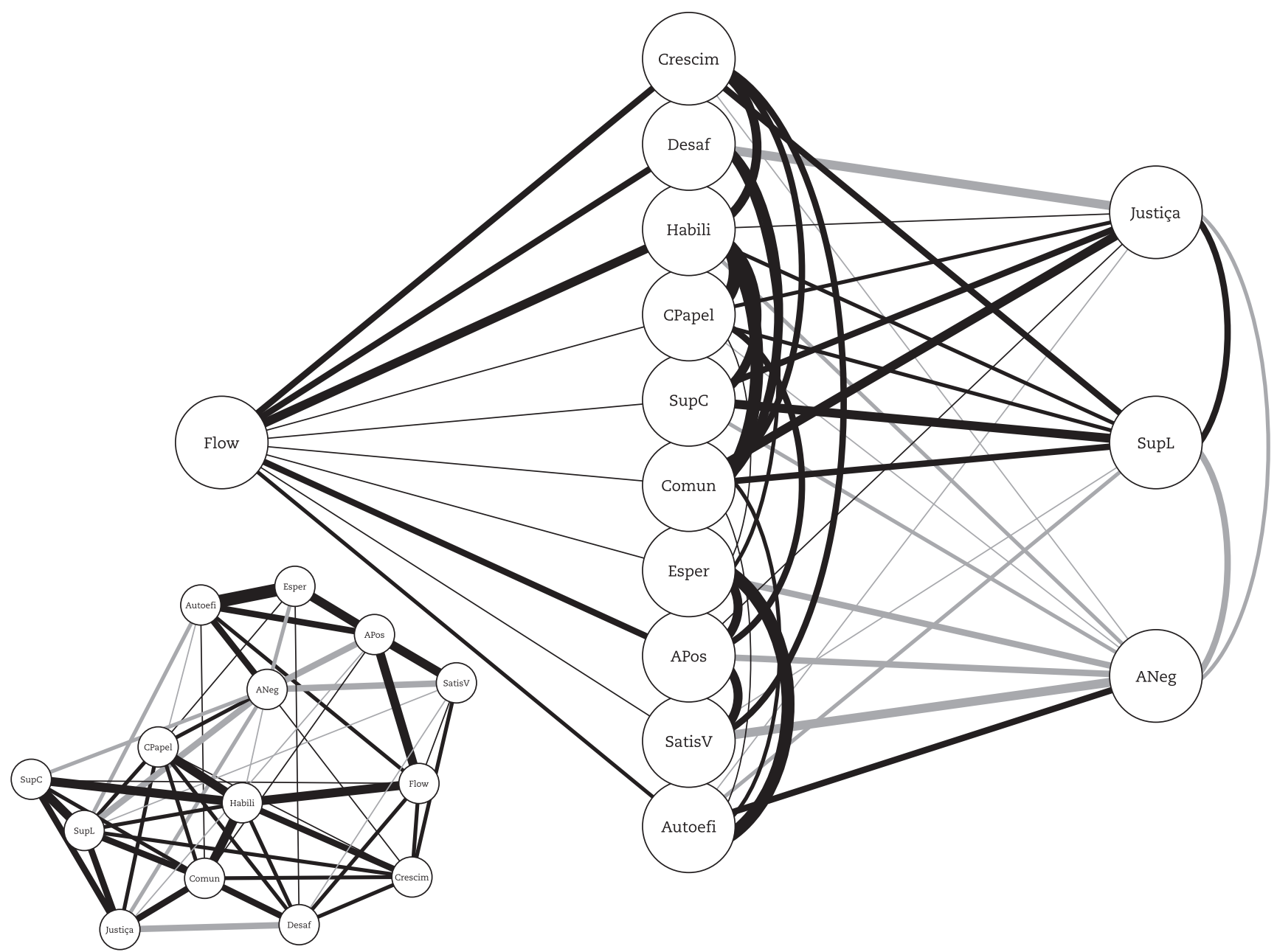

Figura 1. Rede de correlações parciais entre as variáveis flow laboral, Recursos e Demandas de Trabalho e Recursos Individuais.

Layout original: figura menor; flowchart: figura maior. Flow=Flow no Trabalho. Recursos e Demandas de Trabalho: Comum=Comunicação; $\mathrm{CPapel=Clareza}$ de Papel; Crescim=Perspectiva de Crescimento; Desaf=Desafios; Habili=Alinhamento de Habilidades; Justiça=Justiça no Trabalho; SupC=Suporte dos Colegas; SupL = Suporte do Líder. Recursos Individuais: Autoefi=Autoeficácia; Esper=Esperança; NegA=Afetos Negativos; PosA=Afetos Positivos; SatisV=Satisfação com a Vida 
A Figura 2 apresenta o gráfico de fluxo e a análise de rede entre engajamento, recursos e demandas de trabalho, e recursos individuais. O engajamento aparece fortemente associado com as variáveis alinhamento de habilidades e desafios e, não tão intensamente, mas também diretamente associado, a perspectiva de crescimento (Figura 2). No que diz respeito aos recursos individuais, o engajamento associa-se direta e positivamente com os afetos positivos e autoeficácia. Engajamento associou-se indiretamente com comunicação, sendo sua relação intermediada principalmente pelas variáveis alinhamento de habilidades, desafios, e perspectiva de crescimento. Suporte dos colegas associou-se indiretamente ao engajamento principalmente por intermédio da variável alinhamento de habilidades. Também suporte dos colegas teve associação positiva e de alta magnitude com suporte do líder.

De acordo com a análise de influência esperada, apresentada na Figura 3, é possível observar que os nodos alinhamento de habilidades e comunicação possuem alta probabilidade de influenciar positivamente os nodos adjacentes. De maneira inversa, os afetos negativos possuem uma maior probabilidade de influenciar negativamente os nodos adjacentes. Percebe-se que engajamento é a terceira variável mais influenciadora de seu sistema, enquanto flow aparece como a sétima variável mais influente em seu modelo. Ainda, os demais recursos de trabalho e os recursos individuais situam-se em pontos semelhantes em ambas as análises (perto do ponto médio, indicando pouca influência no sistema).

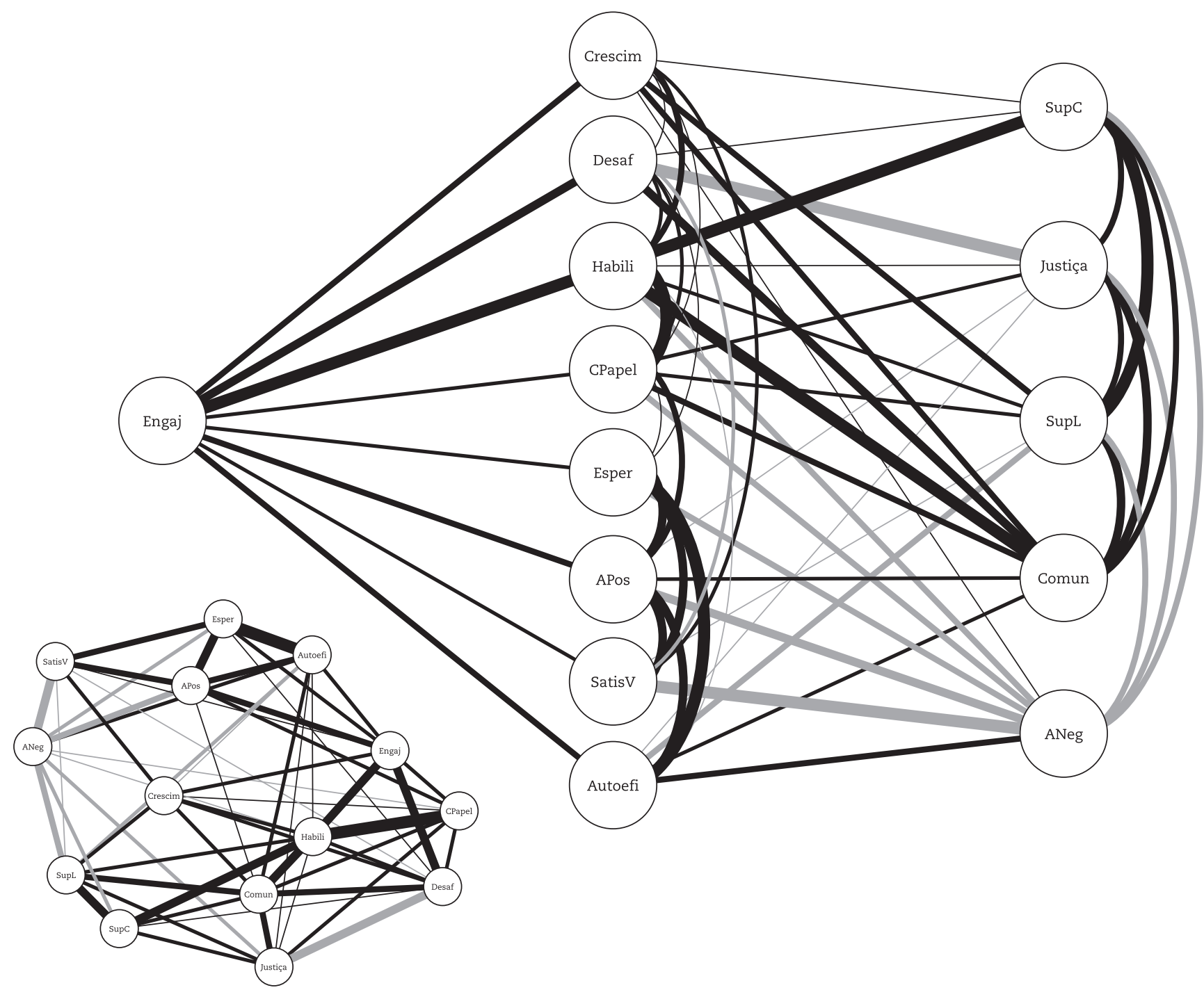

Figura 2. Rede entre engajamento laboral, Recursos e

Demandas de Trabalho e Recursos Individuais.

Engaj=Engajamento no trabalho. Recursos e Demandas de Trabalho: Comum=Comunicação; CPapel=Clareza de Papel; Crescim=Perspectiva de Crescimento; Desaf=Desafios; Habili=Alinhamento de Habilidades; Justiça=Justiça no Trabalho; SupC=Suporte dos Colegas; SupL=Suporte do Líder. Recursos Individuais: Autoefi=Autoeficácia; Esper=Esperança; NegA=Afetos Negativos; PosA=Afetos Positivos; SatisV=Satisfação com a Vida 


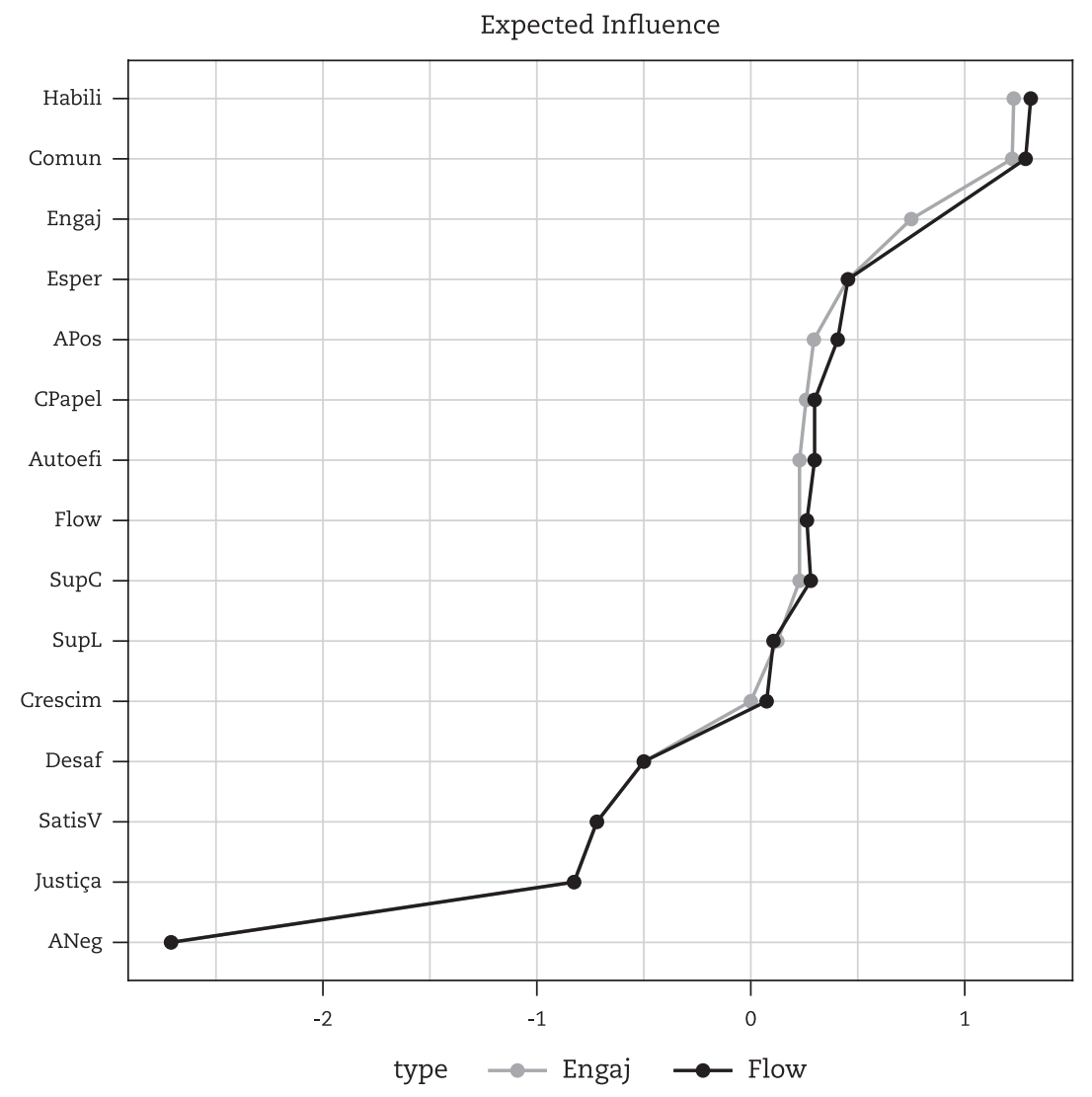

Figura 3. Escores padronizados de influência esperada de cada vértice do grafo de engajamento laboral e flow laboral, com Recursos e Demandas de Trabalho, e Recursos Individuais

\begin{abstract}
Engaj=Engajamento no trabalho; Flow=Flow no trabalho. Recursos e Demandas de Trabalho: Comum=Comunicação; CPapel=Clareza de Papel; Crescim=Perspectiva de Crescimento; Desaf=Desafios; Habili=Alinhamento de Habilidades; Justiça=Justiça no Trabalho; SupC=Suporte dos Colegas; SupL=Suporte do Líder. Recursos Individuais: Autoefi=Autoeficácia; Esper=Esperança; NegA=Afetos Negativos; PosA=Afetos Positivos; SatisV=Satisfação com a Vida.
\end{abstract}

\section{Discussão}

Observando-se os resultados da análise de rede do flow com recursos de trabalho e com recursos individuais, é possível perceber a relevância dos nodos alinhamento de habilidades e afetos positivos. Sabe-se que para a experiência de flow é importante tanto estar absorvido em uma tarefa que estimule habilidades já existentes quanto estar gostando do que se faz naquele momento, característica chamada de envolvimento pela escala WOLF (Bakker, 2008; Vazquez et al., 2015). Essa definição parece explicar a associação positiva e de alta magnitude entre flow - alinhamento de habilidades e flow - afetos positivos.

Ao mesmo tempo, o nodo alinhamento de habilidades está associado com magnitude alta e positiva à clareza de papel. Assim, supõe-se a partir desses resultados, que uma equipe com papéis bem estabelecidos e alinhados às habilidades de cada trabalhador possuirá mais facilidade de entrar em flow. Do mesmo modo, como não se sabe a direcionalidade da associação entre as variáveis, pode-se levantar a hipótese de que uma equipe que entre em flow com maior frequência poderia mais facilmente alinhar suas habilidades às tarefas exercidas e demarcar papéis nítidos entre si.

No que concerne aos afetos positivos - a segunda maior associação direta de flow - verifica-se na Figura 1 a sua associação positiva de alta magnitude com esperança e satisfação com a vida. Em consonância com Salanova et al. (2006), os recursos de trabalho quando estão em conjunto mantêm relações recíprocas com o estado de flow laboral. Em tais pesquisas, é apontada uma espiral de ganho, em que os afetos positivos contribuem para o desenvolvimento dos recursos individuais e laborais, facilitando a vivência de flow. Igualmente, o flow alimenta os estados de bem-estar, facilita a criação de recursos e estimula emoções positivas. Portanto, experiências positivas em relação ao trabalho podem gerar a criação de recursos que, por sua vez, influenciam na vivência de flow, e ambos funcionam em retroalimentação (Salanova et al., 2006). Da mesma forma, oflow laboral possui associação 
de segundo grau com os afetos negativos; afetos negativos possui associação negativa de alta magnitude com o nodo afetos positivos. Isso pode denotar a importância de se manter os afetos negativos baixos em um ambiente de trabalho para que se facilite a vivência de flow.

Verificam-se nos resultados da rede entre engajamento, recursos e demandas de trabalho, e recursos individuais, associações diretas e de alta magnitude entre engajamento e alinhamento de habilidades, desafios, perspectiva de crescimento e afetos positivos. As associações positivas e diretas com desafios e perspectiva de crescimento apontam para o caráter de investimento de energia e enfrentamento das dificuldades apresentado pelo engajamento nas atividades laborais (Vazquez et al., 2015). Disso, pode-se inferir que um funcionário com alto engajamento será apresentado tanto a maiores desafios quanto a um maior número de oportunidades para desenvolver suas competências. Ainda, por não se saber a direcionalidade das associações na Figura 2, pode-se supor que o engajamento é promovido ao se possibilitar oportunidades de crescimento e desafios adequados aos colaboradores.

Na Figura 2, engajamento também se associou direta e positivamente com as variáveis esperança e autoeficácia (as quais tiveram entre si a associação positiva de maior magnitude da rede). Os autores Uusiautti e Määttä (2015) propõem que as expectativas positivas em relação ao futuro podem resultar em "comportamentos esperançosos" e, assim, estar relacionada ao engajamento laboral. Sobretudo, é interessante sublinhar-se que a relação entre engajamento e esperança aparece como sendo recíproca, tanto na atual pesquisa como nos resultados de Uusiautti e Määttä (2015). Assim como a esperança, Consiglio, Borgogni, Di Tecco e Schaufeli, (2016) verificaram que trabalhadores autoeficazes podem conseguir interferir de maneira benéfica em seu entorno, exercendo controle sobre as relações sociais e, assim, desenvolvendo um clima positivo juntamente aos demais e sua liderança. Essa afirmação encontra respaldo nos resultados do presente estudo ao se observar a associação de alta magnitude entre as variáveis afetos positivos, autoeficácia, esperança e satisfação com a vida.

A análise de influência esperada, apresentada na Figura 3, evidencia o papel das variáveis alinhamento de habilidades, comunicação, e afetos negativos. Alinhamento de habilidades é um recurso de trabalho que aborda a percepção do trabalhador sobre suas competências e possibilidades de aprendizagem em um cargo (Farina et al., no prelo). Exemplo de um item desse fator pode ser: "Meu trabalho atual combina bem com o que posso realizar.". A variável comunicação também apresentou alta influência esperada no modelo. Tal variável retrata a percepção do colaborador de que é bem informado sobre o que ocorre na sua organização/equipe (Farina et al., no prelo). Exemplo de item: "Sou informado adequadamente dos desenvolvimentos na minha organização.”. No presente modelo, esses nodos, quando ativados, possuíram as maiores probabilidades de ativarem seus nodos adjacentes. Portanto, as variáveis alinhamento de habilidades e comunicação são indicadas como possíveis focos de intervenções que visem promover engajamento e flow no trabalho.

Por outro lado, a alta influência esperada da variável afetos negativos ressalta a importância de manter-se um ambiente laboral seguro e estável. Isto pois, no atual modelo, quanto mais ativado, maior a probabilidade deste nodo desativar os nodos adjacentes. Ademais, na Figura 3 percebe-se que engajamento teve a terceira maior influência esperada em seu modelo. Flow, por outro lado, localizou-se perto do ponto médio, indicando pouca influência. Isto condiz com as próprias definições dos construtos. Enquanto engajamento é um estado disposicional que depende e está associado aos recursos e às demandas de trabalho, flow é um estado momentâneo de alta concentração, prazer e produtividade.

Esta pesquisa procurou investigar as associações entre engajamento e flow laboral com recursos individuais e recursos de trabalho. Partiu-se do Modelo RDT e utilizaram-se análises de rede. De modo geral, os resultados levam a pensar em associações de predição mútuas, que podem se modificar de uma para a outra de acordo com o contexto em que estão inseridas. De acordo com este estudo, para a promoção de flow e de engajamento, a autoeficácia e os afetos positivos são recursos individuais que merecem destaque no ambiente laboral.

Ainda, o presente estudo evidenciou três variáveis como importantes influenciadoras no modelo analisado. São elas: alinhamento de habilidades, comunicação e afetos negativos. A partir dos resultados encontrados, levanta-se a hipótese de que as duas primeiras sejam dois recursos de trabalho com a maior probabilidade de, quando estimulados, potencializar os demais construtos investigados. Por outro lado, a variável afetos negativos possui a maior probabilidade, quando intensificados, de desativar as demais variáveis investigadas.

Tem-se como limitações desta pesquisa a amostra composta majoritariamente por pós-graduados, o que pode enviesar os resultados aqui encontrados. A fim de averiguar a estabilidade do modelo aqui apresentado, sugere-se que futuros estudos continuem a investigar essas questões para que seja possível um maior aprofundamento nos resultados desta produção científica. Por fim, considerando-se as evidências encontradas, os resultados propõem que intervenções para a promoção de flow e de engajamento no trabalho que considerarem a constelação de variáveis no âmbito de uma organização tendem a ser mais eficazes. Cabe ressaltar que este estudo também contribui para a ampliação do entendimento do funcionamento do flow e do engajamento no trabalho de forma diferenciada de outras pesquisas, na medida em que se utiliza de diversas variáveis pessoais e de contexto ao mesmo tempo - o que, até então, ainda não foi encontrado na literatura existente (Farina et al., 2018). 


\section{Referências}

Bakker, A. B. (2008). The work-related flow inventory: Construction and initial validation of the WOLF. Journal of Vocational Behavior, 72(3), 400-414. doi: 10.1016/j.jvb.2007.11.007

Consiglio, C., Borgogni, L., Di Tecco, C., \& Schaufeli, W. B. (2016). What makes employees engaged with their work? The role of selfefficacy and employee's perceptions of social context over time. Career Development International, 21(2), 125-143. doi: 10.1108/CDI03-2015-0045

Cousins, R., Mackay, C. J., Clarke, S. D., Kelly, C., Kelly, P. J., \& McCaig, R. H. (2004). 'Management standards' work-related stress in the UK: Practical development. Work \& Stress, 18(2), 113-136. doi: 10.1080/02678370 410001734322

Csikszentmihalyi, M. (1999). If we are so rich, why aren't we happy? American Psychologist Association, 54(10), 821-827. doi: 10.1037/0003066X.54.10.821

Datu, J. A. D., \& Mateo, N. J. (2015). Work-related flow dimensions differentially predict anxiety, life satisfaction, and work longevity among filipino counselors. Current Psychology, 36(2), 203-208. doi: 10.1007/s12144-015-9401-3

Epskamp, S., Cramer, A. O. J, Waldorp, L. J., Schmittmann, V. D., Borsboom, D. (2012). qgraph: Network Visualizations of Relationships in Psychometric Data. Journal of Statistical Software, 48(4), 1-18. doi: 10.18637/jss.v048.i04

Epskamp, S., \& Fried, E. I. (2018). A tutorial on regularized partial correlation networks. Psychological Methods, 23(4), 617-634. doi: 10.1037/ met0000167

Farina, L. S. A., Rodrigues, G. R., Fagundes, N. K., Carafini, T. C., Moreira, L. G. C. S., Machado, W. L., Hutz, C. S. (no prelo). Construção e Evidências de Validade do Questionário de Recursos e Demandas Laborais. Avaliação Psicológica, Manuscrito aceito para publicação.

Farina, L. S. A., Rodrigues, G. R., \& Hutz, C. S. (2018). Flow e engajamento no trabalho: Uma revisão de literatura. Psico-USF, 23(4), 633642. doi: $10.1590 / 1413-82712018230404$

Foygel, R., \& Drton, M. (2010). Extended bayesian information criteria for gaussian graphical models. Proceedings of the 23rd International Conference on Neural Information Processing Systems, 604-612. Recuperado de arxiv.org/pdf/1011.6640.pdf

Freitas, C. P. P., Damásio, B. F., \& Koller, S. H. (2019). Work-related flow inventory: Evidence of validity of the brazilian version. Paidéia (Ribeirão Preto), 29(e2901), 1-8. doi: 10.1590/1982-4327e2901

Friedman, J., Hastie, T., \& Tibshirani, R. (2008). Sparse inverse covariance estimation with the graphical lasso. Biostatistics, 9(3), $432-441$. doi:10.1093/biostatistics/kxm045

Hakanen, J. J., \& Schaufeli, W. B. (2012). Do burnout and work engagement predict depressive symptoms and life satisfaction ? A threewave seven-year prospective study. Journal of Affective Disorders, 141(2-3), 415-424. doi: 10.1016/j.jad.2012.02.043

Hutz, C. S., Zanon, C., \& Bardagi, M. P. (2014). Satisfação de Vida. Em C. S. Hutz (Ed.), Avaliação em Psicologia Positiva (pp. 43-47). Porto Alegre: Artmed.

Machado, W. L., Vissoci, J., \& Epskamp, S. (2015). Análise de rede aplicada à Psicometria e à Avaliação Psicológica. Em C. S. Hutz, D. R. Bandeira \& C. M. Trentini (Eds.). Psicometria (pp. 125-144). Porto Alegre: Artmed.

Mäkikangas, A., Bakker, A. B., Aunola, K., \& Demerouti, E. (2010). Job resources and flow at work: Modelling the relationship via latent growth curve and mixture model methodology. Journal of Occupational and Organizational Psychology, 83(3), 795-814. doi: 10.1348/096317909X476333

Nielsen, K., \& Cleal, B. (2010). Predicting flow at work: Investigating the activities and job characteristics that predict flow states at work. Journal of Occupational Health Psychology, 15(2), 180-190. doi: 10.1037/a0018893

Pacico, J. C., Ferraz, S. B., \& Hutz, C. S. (2014). Autoeficácia - Yes we can! Em C. S. Hutz (Ed.), Avaliação em Psicologia Positiva (pp. 111-119). Porto Alegre: Artmed.

Pacico, J. C., Zanon, C., Bastianello, M. R., Reppold, C. T., \& Hutz, C. S. (2013). Adaptation and validation of the Brazilian version of the Hope Index. International Journal of Testing, 13(3), 193-200. doi: 10.1080/15305058.2012.664833

Robinaugh, D. J., Millner, A. J., \& McNally, R. J. (2016). Identifying highly influential nodes in the complicated grief network. Journal of Abnormal Psychology, 125(6), 747-757. doi: 10.1037/abn0000181

RStudio (2012). RStudio: Integrated development environment for R (Versão 1.2.1335) [Software de Computador]. Boston, MA.

Salanova, M., Bakker, A. B., \& Llorens, S. (2006). Flow at work: evidence for an upward spiral of personal and organizational resources. Journal of Happiness Studies, 7(1), 1-22. doi: 10.1007/s10902-005-8854-8

Schaufeli, W., Bakker, A. B., \& Rhenen, W. V. (2009). How changes in job demands and resources predict burnout, work engagement, and sickness absenteeism. Journal of Organizational Behavior, 30(7), 893-917. doi: 10.1002/job.595

Schaufeli, W. (2013). What is Engagement? Em C. Truss, R. Delbridge, K. Alfes, A. Shantz \& E. Soane (Eds.). Employee engagement in theory and practice (pp. 15-35). London: Routledge.

Schaufeli, W., Dijikstra, P., \& Vazquez, A. C. (2013). Engajamento no Trabalho. São Paulo: Casa do Psicólogo.

Schmittmann, V. D., Cramer, A. O. J., Waldorp, L. J., Epskamp, S., Kievit, R. A., \& Borsboom, D. (2013). Deconstructing the construct: A network perspective on psychological phenomena. New Ideas in Psychology, 31(1), 43-53. doi: 10.1016/j.newideapsych.2011.02.007

Taris, T. W., \& Schaufeli, W. B. (2016). The job demands-resources model. Em S. Clarke, T. M. Probst, F. Guldenmund \& J. Passmore (Eds.). The Wiley Blackwell handbook of the psychology of occupational safety and workplace health (pp. 157-180). Chichester: John Wiley.

Uusiautti, S., \& Määttä, K. (2015). Engagement and hope as factors behind success at work. Journal of Studies in Education, 5(2), 106-118. doi: 10.5296/jse.v5i2.7434

Vazquez, A. C. S., Magnan, E. S., Pacico, J. C., Hutz, C. S., \& Schaufeli, W. B. (2015). Adaptation and validation of the brazilian version of the Utrecht Work Engagement Scale. Psico-USF, 20(2), 207-217. doi: 10.1590/1413-82712015200202

Zanon, C., Bardagi, M. P., Layous, K., \& Hutz, C. S. (2014). Validation of the satisfaction with life scale to brazilians: Evidences of measurement non invariance across brazil and US. Social Indicators Research, 119(1), 443-453. doi 10.1007/s11205-013-0478-5 
Zanon, C., Bastianello, M. R., Pacico, J. C., \& Hutz, C. S. (2013). Desenvolvimento e validação de uma escala de afetos positivos e negativos. Psico-USF, 18(2), 193-202. doi: 10.1590/S1413-82712013000200003

Zubair, A., \& Kamal, A. (2015). Work related flow, psychological capital, and creativity among employees of software houses. Psychological Studies, 60(3), 321-331. doi: 10.1007/s12646-015-0330-x30-x

\section{Sobre os autores}

Larissa Sanford Ayres Farina é psicóloga, formada pela Universidade Federal do Rio Grande do Sul (UFRGS), especialista em Gestão de Pessoas e em Dinâmica dos Grupos, mestra e atual doutoranda do Programa de Pós-Graduação em Psicologia também pela UFRGS.

Gabriel dos Reis Rodrigues é graduando em Psicologia pela Universidade Federal do Rio Grande do Sul (UFRGS) e, atualmente, é bolsista de Iniciação Científica no Laboratório de Mensuração (Instituto de Psicologia - UFRGS).

Natália Kluwe Fagundes é psicóloga pela Universidade Federal do Rio Grande do Sul (UFRGS), tendo atuado como bolsista de Iniciação Científica no Laboratório de Mensuração (Instituto de Psicologia - UFRGS).

Thamires Casarotto Carafini é graduanda na Universidade Luterana do Brasil no curso de Psicologia e bolsista voluntária de Iniciação Científica no Laboratório de Mensuração (Instituto de Psicologia - UFRGS).

Laisla Gabriele Conceição Soares Moreira é graduanda em Psicologia pela Faculdade de Desenvolvimento do Rio Grande do Sul e bolsista voluntária de Iniciação Científica no Laboratório de Mensuração (Instituto de Psicologia - UFRGS).

Wagner De Lara Machado é psicólogo com pós-doutorado como consultor de análise quantitativa de dados e psicometria (UFRGS) e professor titular na PUCRS, coordenador do Grupo de Pesquisa de Avaliação em Bem-estar e Saúde Mental.

Claudio Simon Hutz é psicólogo com doutorado na University of Iowa (USA) e pós-doutorado na Arizona State University (USA). É professor titular da UFRGS e coordenador do Laboratório de Mensuração (Instituto de Psicologia - UFRGS). 